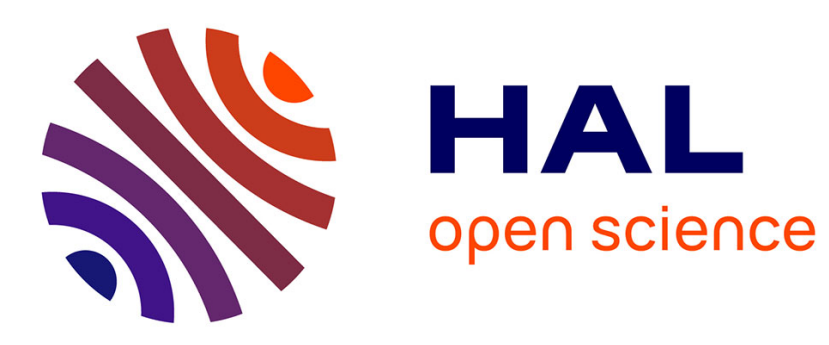

\title{
Spatial optimal disturbances in swept attachment-line boundary layers
}

\author{
Alan Guégan, Peter J. Schmid, Patrick Huerre
}

\section{To cite this version:}

Alan Guégan, Peter J. Schmid, Patrick Huerre. Spatial optimal disturbances in swept attachment-line boundary layers. Journal of Fluid Mechanics, 2008, 603 (mai), pp.179-188. 10.1017/s0022112008001067 . hal-01022808

\section{HAL Id: hal-01022808 \\ https://hal-polytechnique.archives-ouvertes.fr/hal-01022808}

Submitted on $20 \mathrm{Jul} 2014$

HAL is a multi-disciplinary open access archive for the deposit and dissemination of scientific research documents, whether they are published or not. The documents may come from teaching and research institutions in France or abroad, or from public or private research centers.
L'archive ouverte pluridisciplinaire HAL, est destinée au dépôt et à la diffusion de documents scientifiques de niveau recherche, publiés ou non, émanant des établissements d'enseignement et de recherche français ou étrangers, des laboratoires publics ou privés. 


\title{
Spatial optimal disturbances in swept attachment-line boundary layers
}

\author{
ALAN GUÉGAN, PETER J. SCHMID \\ AND PATRICK HUERRE \\ Laboratoire d'Hydrodynamique (LadHyX), \\ CNRS-École Polytechnique, F-91128 Palaiseau, France
}

(Received 24 October 2007 and in revised form 26 February 2008)

\begin{abstract}
A formulation based on direct and adjoint parabolized equations is developed to account for the spatial evolution of perturbations in swept attachment-line boundary layers. For sweep Reynolds numbers larger than $\mathrm{Re}=100$ the dynamics is dominated by a lift-up mechanism which is responsible for large energy amplification by transforming spanwise vortices into spanwise streaks. This mechanism favours steady perturbations with a chordwise scale that quantitatively matches its counterpart for classical Blasius boundary layers.
\end{abstract}

\section{Introduction}

Swept attachment-line boundary layers count among the most common flow configurations in aero- and hydrodynamical applications as they form on any moving, yawed, blunt-edged body that is exposed to the surrounding fluid. The swept wing of an aircraft is the most obvious case where attachment-line boundary layers can be observed, but other applications abound, ranging from rudders or control surfaces of naval or submarine vessels to catenary riser cables, from hypersonic re-entry vehicles to wings of soaring birds.

The prominence of this type of flow as well as its important role in numerous applications have fuelled a great many studies on the flow behaviour and perturbation dynamics of swept attachment-line boundary layers. Early studies have focused on experimental efforts (see e.g. Gregory 1960; Gaster 1967; Cumpsty \& Head 1969; Pfenninger \& Bacon 1969; Pfenninger 1977) in an attempt to analyse and, ultimately, prevent transition downstream of the stagnation line due to inherent instabilities. These studies were followed by theoretical investigations into the stability of flow in the vicinity of the attachment line. Hall, Malik \& Poll (1984) determined a critical Reynolds number (and thus sweep angle) for the appearance of a modal temporal instability under the Görtler-Hämmerlin assumption (see Görtler 1955; Hämmerlin 1955). These computations were later extended to more general flow perturbations by Lin \& Malik (1996) but no further modal instabilities have been found beyond the Görtler-Hämmerlin type. Direct numerical simulations (see e.g. Joslin 1995; Theofilis 1998) have confirmed these findings for the supercritical regime; the subcritical case, however, proved more difficult. The potential for temporal amplification of disturbance energy in the subcritical regime has been investigated by Obrist \& Schmid (2003b) using a non-modal stability analysis and by Guégan, Schmid \& Huerre (2006) and Guégan, Huerre \& Schmid (2007) using a variational formulation based on the direct and adjoint linearized Navier-Stokes equations. In both cases, large transient growth 


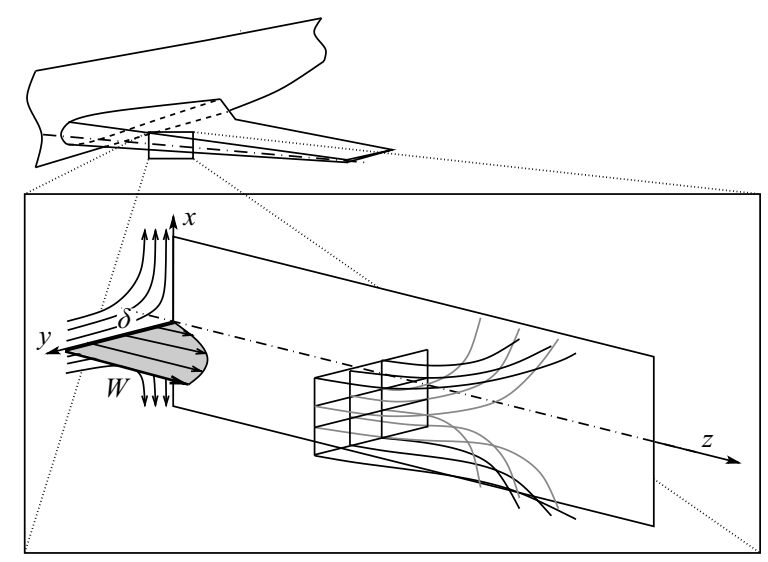

FIGURE 1. Sketch of geometry.

of disturbance energy has been found, providing evidence that short-term temporal instability mechanisms may play an important role in the transition process.

The stability problem of attachment-line boundary layers has also been treated within a spatial framework. In this case, the response to a harmonic disturbance source is followed downstream (and upstream) of the location of origin, and growth/decay of disturbance size is described in space which, mathematically, leads to a signalling problem. This type of analysis, though closer to realistic configurations, poses great challenges to the computation of stability characteristics as well as to their interpretation. Recently, the spatial spectrum has been computed by Theofilis (1995), and its link to the absolute/convective stability behaviour has been investigated by Turkyilmazog̃lu \& Gajjar (1999).

Recalling the potential for large transient growth within a temporal framework (Obrist \& Schmid 2003b; Guégan et al. 2006, 2007), it seems plausible to envision the presence of non-modal effects also in a spatial setting. The spatial nonmodal stability problem is the topic of this article. In particular, we are interested in the shape and evolution of disturbances that are most favoured by the spatial initialvalue problem. To this end, an optimization scheme based on the direct and adjoint spatial equations is developed and applied. Any spatial non-modal stability analysis, however, has to deal with the inherent ellipticity of the resulting signalling problem. The most widely used method consists of the direct elimination of elliptic terms (contained in the viscous and pressure terms) by scaling arguments (see Bertolotti, Herbert \& Spalart 1992; Chang \& Malik 1993). In our case, we can take advantage of the strong convective nature of the flow in the spanwise direction due to the sweep velocity and argue (by simple scaling) for the elimination of subdominant elliptic components of the governing equations. With this judicious simplification, the direct-adjoint system can be solved efficiently by a standard marching technique.

\section{Mathematical model}

For the flow in the vicinity of the swept attachment line we will choose the model of swept Hiemenz flow as already introduced in, for example, Guégan et al. (2006) to analyse the spatial evolution of disturbances of small amplitudes. The coordinates $(x, y, z)$ refer to the chordwise, wall-normal and spanwise directions, respectively, with $(u, v, w)$ the corresponding velocity components (see figure 1). 
In swept Hiemenz flow the free-stream spanwise sweep velocity $W_{\infty}$ is commonly taken as the reference velocity. After normalization by $W_{\infty}$ the chordwise basic velocity component $x U$ scales like $x / R e$ whereas the spanwise basic velocity component $W$ is of order unity. Consequently, at Reynolds numbers higher than about $10^{2}$ and chordwise $x$-distances from the attachment line of the order of the boundary-layer thickness $\delta \approx 3$, the basic sweep velocity $W$ is more than a hundred times larger than the chordwise basic velocity. The flow is thus strongly advective in the spanwise direction.

\subsection{Parabolized governing equations}

Within the boundary layer of thickness $\delta$ and close to the attachment line the typical length scales are $(x, y, z) \sim(\delta, \delta, 1)$ which, by invoking the continuity equation, implies that the velocity field behaves as $(u, v, w) \sim(\delta, \delta, 1)$. Based on these scalings we conclude that derivative terms with respect to the spanwise $z$-direction are negligible when compared with derivatives in the remaining two directions. This simple scaling argument justifies discarding second-order terms of the linearized Navier-Stokes equations in the spanwise $z$-direction as well as neglecting the spanwise pressure gradient. As a result one obtains the parabolized evolution equations for perturbations superimposed on swept Hiemenz flow. Further assuming a time-harmonic form of the perturbations and introducing a temporal circular frequency $\omega$, we arrive at the governing equations

$$
\begin{aligned}
W \frac{\partial u}{\partial z} & =\mathscr{L} u-U u-x U^{\prime} v-\frac{\partial p}{\partial x}, \\
W \frac{\partial v}{\partial z} & =\mathscr{L} v-V^{\prime} v-\frac{\partial p}{\partial y}, \\
W \frac{\partial w}{\partial z} & =\mathscr{L} w-V^{\prime} w \\
0 & =\frac{\partial u}{\partial x}+\frac{\partial v}{\partial y}+\frac{\partial w}{\partial z},
\end{aligned}
$$

with $\mathscr{L}=\mathrm{i} \omega-x U \partial / \partial x-V \partial / \partial y+\left(\partial^{2} / \partial x^{2}+\partial^{2} / \partial y^{2}\right) / R e$. This simplified set of equations will form the basis for exploring the spatial amplification of energy in swept Hiemenz flow and for computing the flow structures that are most favoured by a non-normal spatial evolution process. To this end, an objective functional has to be defined which will subsequently be maximized while satisfying the governing equations (2.1) via an adjoint set of equations.

\subsection{Objective functional, adjoint equations and optimality conditions}

The relative amplification of the disturbance energy between the spanwise location $z=0$ and a prescribed spanwise distance $z=z_{\max }$ will be taken as a measure of disturbance growth. The objective functional $\mathscr{I}$ to be maximized is then

$$
\mathscr{I} \equiv \frac{E\left(z=z_{\max }\right)}{E(z=0)} \quad \text { with } \quad E(z)=\frac{1}{2} \int_{y \geqslant 0} \int_{-\infty}^{\infty} \lambda_{E}(x)\left(u^{2}+v^{2}+R e^{2} w^{2}\right) \mathrm{d} x \mathrm{~d} y .
$$

The $R e^{2}$-factor in front of the $w$-component has been introduced to account for the scaling of velocities $(u, v, w)$. The Gaussian weight function $\lambda_{E}(x)=\mathrm{e}^{-(x / L)^{2}}$ in the energy integral is needed to avoid divergence in the subsequent optimization process. The divergence is due to the fact that infinite amplification factors could be 
obtained by exploiting the unbounded, thus unphysical, nature of the basic velocity field as $x \rightarrow \pm \infty$. We will explore the dependence of the results on the choice of the characteristic cutoff length $L$ in the later part of this study.

The maximum of the objective functional $\mathscr{I}$ is computed via a direct-adjoint formulation of the optimization problem followed by an iterative scheme based on the computed gradient information. The adjoint equations can easily be obtained by a variational formulation (see e.g. Guégan et al. 2006) and are

$$
\begin{aligned}
W \frac{\partial \tilde{u}}{\partial z} & =\mathscr{M} \tilde{u}+U \tilde{u}-\frac{\partial \tilde{p}}{\partial x}, \\
W \frac{\partial \tilde{v}}{\partial z} & =\mathscr{M} \tilde{v}+x U^{\prime} \tilde{u}+V^{\prime} \tilde{v}+W^{\prime} \tilde{w}-\frac{\partial \tilde{p}}{\partial y}, \\
W \frac{\partial \tilde{w}}{\partial z} & =\mathscr{M} \tilde{w}-\frac{\partial \tilde{p}}{\partial z}, \\
0 & =\frac{\partial \tilde{u}}{\partial x}+\frac{\partial \tilde{v}}{\partial y},
\end{aligned}
$$

with $\mathscr{M}=\mathrm{i} \omega-x U \partial / \partial x-V \partial / \partial y-U-V^{\prime}-\left(\partial^{2} / \partial x^{2}+\partial^{2} / \partial y^{2}\right) / R e$. According to the standard direct-adjoint formulation, the set of adjoint equations has to be integrated in the upstream spanwise $z$-direction. For this reason, terminal conditions at $z=z_{\max }$ have to be specified for the adjoint variables. The variational formalism provides the following conditions:

$$
\begin{aligned}
W \tilde{u}\left(z_{\max }\right) & =\frac{2}{E_{0}} \lambda_{E} u\left(z_{\max }\right), \\
W \tilde{v}\left(z_{\max }\right) & =\frac{2}{E_{0}} \lambda_{E} v\left(z_{\max }\right), \\
W \tilde{w}\left(z_{\max }\right)+\tilde{p}\left(z_{\max }\right) & =\frac{2}{E_{0}} \operatorname{Re}^{2} \lambda_{E} w\left(z_{\max }\right) .
\end{aligned}
$$

The gradient of the objective functional $\mathscr{I}$ with respect to the initial conditions $\left(u_{0}, v_{0}, w_{0}\right)$ follows as

$$
\begin{aligned}
& \nabla_{u_{0}} \mathscr{I}=-\frac{2 E\left(z_{\max }\right)}{E_{0}^{2}} \lambda_{E} u_{0}+W \tilde{u}(0), \\
& \nabla_{v_{0}} \mathscr{I}=-\frac{2 E\left(z_{\max }\right)}{E_{0}^{2}} \lambda_{E} v_{0}+W \tilde{v}(0), \\
& \nabla_{w_{0}} \mathscr{I}=-\frac{2 E\left(z_{\max }\right)}{E_{0}^{2}} R^{2} \lambda_{E} w_{0}+W \tilde{w}(0)+\tilde{p}(0) .
\end{aligned}
$$

The system of equations (2.1)-(2.5) is then used as follows. Starting with spatial initial conditions at $z=0$, the direct set of equations (2.1) is marched forward to $z=z_{\max }$ at which point the direct solution is converted via (2.4) into the terminal condition for the adjoint equations (2.3). The adjoint equations are then integrated backward from $z=z_{\max }$ to $z=0$, after which the gradient of the objective functional with respect to the initial conditions (2.5) can be evaluated. The gradients are then used to update the previously chosen initial condition, and the next iteration commences.

It is important to note that, within the parabolized framework, the direct and adjoint equations display marked differences. Most significantly, in the adjoint problem mass is conserved in $(x, y)$-planes only, since the continuity equation is reduced to 
its two-dimensional form $(2.3 d)$. The reason for this is the absence of a spanwise pressure gradient in the direct spanwise momentum equation (2.1c). In contrast, a spanwise adjoint pressure gradient term is present in the adjoint spanwise momentum equation $(2.3 c)$. The appearance or lack of these terms is a consequence of the parabolization assumption made earlier, and it has implications for the type of numerical method used to solve the above system of equations.

\subsection{Numerical scheme and code validation}

The direct equations (2.1) and adjoint equations (2.3) are similar to the parabolized boundary layer equations used by Andersson, Berggren \& Henningson (1999), Luchini (2000) and Tumin \& Reshotko (2001) to compute optimal spatial disturbances in a Blasius boundary layer, and our numerical scheme has been inspired by these references. The chordwise coupling of our governing equations, however, does not allow the treatment of the $x$-direction by a Fourier transform followed by the solution of a one-dimensional $y$-dependent initial-value problem for each wavenumber; instead, the spatial evolution in $z$ involves the solution of a two-dimensional elliptic problem in $x$ and $y$ at each step. Nevertheless, by rearranging the various terms in the direct and adjoint equations, an efficient numerical scheme can be constructed. We choose a mixed Adams-Bashforth/Crank-Nicolson scheme for the momentum equations and treat the continuity equation as well as the pressure terms implicitly. The adjoint equations are solved with the same marching technique; the additional adjoint pressure term is discretized using an upwind finite-difference stencil. The adjoint terminal conditions are incorporated directly into the right-hand side of the first implicit step of the adjoint equations; by neglecting the chordwise coupling terms over the first step from $z_{\max }$ to $z_{\max }-\Delta z$ a unique solution of the adjoint variables is ensured. The omission of the chordwise coupling terms during the first step of the adjoint equations does not adversely influence the accuracy of the final solution or the effectiveness of the optimization process. For the optimization step the Polak-Ribière conjugate gradient algorithm followed by a line search based on Brent's method is used. Further details of the numerical method can be found in Guégan (2007).

The parabolized equations and the numerical scheme have been validated by considering the special case of optimal perturbations in a parallel and in a spatially growing Blasius boundary layer and comparing the results to Tumin \& Reshotko (2001), Andersson et al. (1999) and Luchini (2000). Despite minor differences in the definition of the objective functional, our results on the maximum spatial energy amplification, the preferred transverse wavenumber and the shape of the optimal perturbation closely matched theirs. This validation exercise provides a stringent test on the accuracy of the numerical technique. Replacing the Blasius velocity profile by the swept Hiemenz base flow, we are now in a position to determine the optimal spatial energy amplification in the vicinity of the attachment line and study the evolution of the spatial optimal perturbations.

\section{Results}

The spatial amplification of perturbation energy depends on four principal parameters, some of them with analogues in standard boundary layers: (i) the Reynolds number $R e=W_{\infty} \delta / v$ measuring, for a constant flow stretching rate $\partial U / \partial x$, the sweep angle of the body or wing; (ii) the non-dimensional frequency $\omega$ of the perturbation; (iii) the parameter $L$ governing the chordwise extent of allowable 


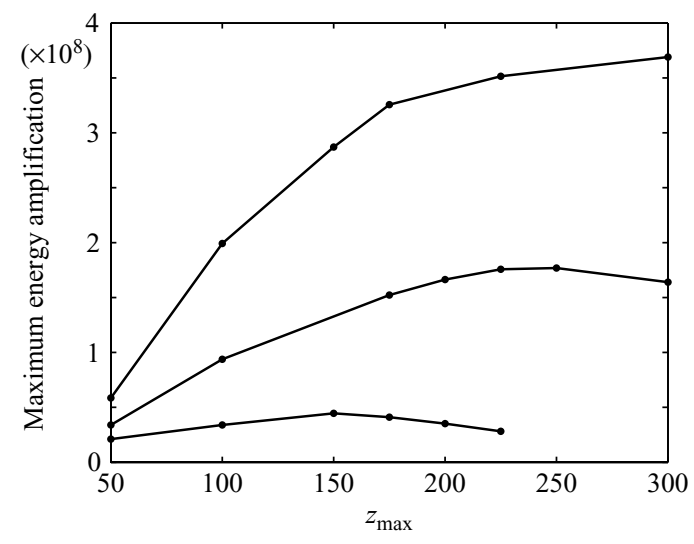

FIGURE 2. Optimal energy amplification versus spanwise optimization distance $z_{\max }$ for Reynolds numbers $R e=400$ (bottom curve), 550 and 700 (top curve). The remaining parameters have been set to $L=9, \omega=0$.

perturbations; this quantity can also be interpreted as providing a cutoff wavenumber; and (iv) the spanwise distance $z_{\max }$ over which the perturbation is allowed to extract energy from the basic flow. The dependence of the optimal spatial energy amplification on these four parameters is assessed in the next section.

\subsection{Optimal spatial energy amplification}

We choose the chordwise parameter as $L=9$, which corresponds to a chordwise extent of the energy weight function of about six times the boundary layer thickness. For a Reynolds number $R e=550$ and a zero frequency $\omega=0$ the perturbation energy is amplified up to $E_{\max }=1.77 \times 10^{8}$ which occurs at $z_{\max }=240$ as shown in figure 2 . Beyond this spanwise location dissipation begins to overcome energy production.

The non-dimensional velocity components $u, v, w$ are of the same order of magnitude which leads, for large Reynolds numbers, to the approximate scaling of the maximum objective functional

$$
\mathscr{I} \approx \operatorname{Re}^{2} \frac{\int_{y \geqslant 0} \int_{-\infty}^{\infty} \lambda_{E}(x) w^{2}\left(z_{\max }\right) \mathrm{d} x \mathrm{~d} y}{\int_{y \geqslant 0} \int_{-\infty}^{\infty} \lambda_{E}(x)\left(u_{0}^{2}+v_{0}^{2}\right) \mathrm{d} x \mathrm{~d} y} .
$$

Note that in (3.1) the optimal initial spanwise velocity $w_{0}$ is found to be identically zero as in classical boundary layers. According to this expression the optimal energy amplification at a given spanwise location $z_{\max }$ is expected to scale as $R e^{2}$ at large Reynolds numbers. Based on a similar reasoning, Luchini (2000) found that the optimal energy amplification in the developing Blasius boundary layer scales linearly with the length Reynolds number. The energy amplification for swept Hiemenz flow is displayed as a function of the Reynolds number Re in figure 3(a) at several spanwise locations $z_{\max }$; the $R e^{2}$-scaling is recovered at higher Reynolds numbers.

The dependence of the optimal energy amplification on the forcing frequency $\omega$ is displayed in figure $3(b)$ for $L=9, z_{\max }=100$ and $R e=550$. As in classical boundary layers, steady disturbances are found to be more amplified than perturbations of non-zero frequency. This behaviour also holds for different Reynolds numbers $R e$ and optimization lengths $z_{\max }$. 

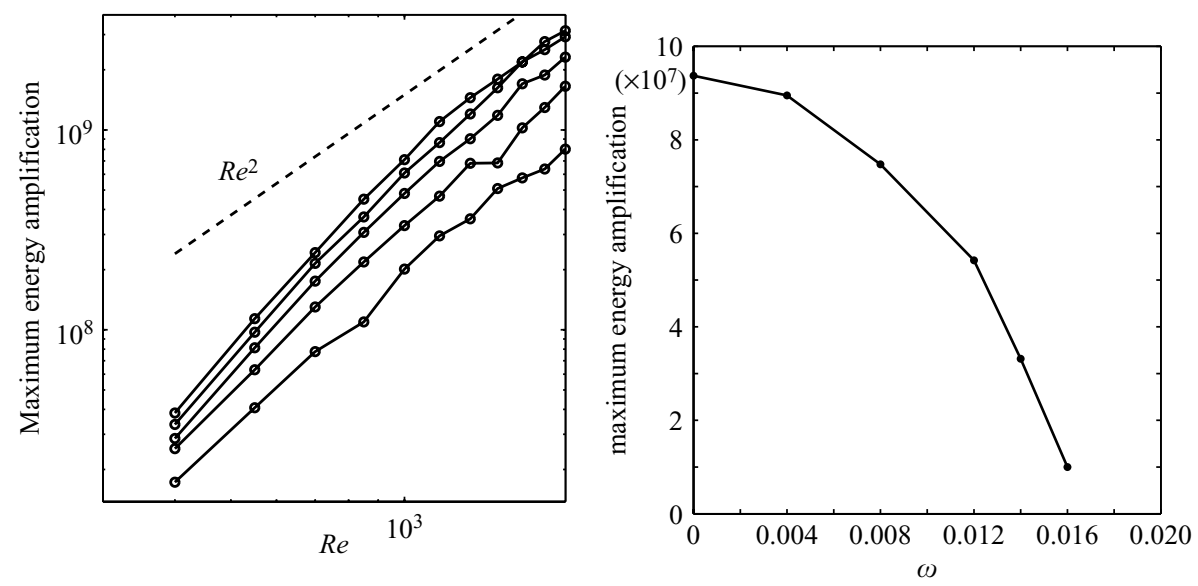

FiguRE 3. (a) Optimal energy amplification versus Reynolds number $R e$ for $z_{\max }=60$ (bottom curve), 80, 100, 120 and 140 (top curve), at $\omega=0$. (b) Maximum energy amplification versus forcing frequency $\omega$ for $L=9, z_{\max }=100$. The Reynolds number has been set to $R e=550$.

\subsection{Optimal spatial disturbances}

Optimal spatial disturbances take the shape of spanwise counter-rotating vortices which develop further downstream into spanwise streaks via the lift-up mechanism (Landahl 1980). The rise of the spanwise $w$-velocity from initial chordwise $u$ - and wall-normal $v$-velocities is displayed in figure 4 .

The isosurfaces of $u$ and $v$ that represent the optimal initial spanwise vortex are seen to terminate prior to reaching the outlet at $z_{\max }$, illustrating that both velocity components are damped to less than $20 \%$ of their initial value between the inlet $z=0$ and the outlet $z=z_{\max }$. By contrast, the $20 \%$-isosurface of the spanwise $w$-velocity expands from $z \approx 1$ to $z=z_{\max }$ confirming the rapid rise of spanwise streaks.

When the chordwise length parameter is set equal to the boundary-layer thickness $L=3$, only three spanwise vortices are formed near the stagnation line. As $L$ is increased, more spanwise rolls of nearly constant width are observed, as shown in figure 5 for $L$ varying from 3 to 12 . The number of spanwise vortices may be estimated by counting the local maxima of the wall-normal $v$-velocity along the chordwise $x$ axis. The number of vortices increases with the chordwise length parameter $L$; the chordwise width of the optimal spanwise vortices, however, only varies slightly with $L$ beyond a spanwise cutoff length of about $L=9$.

The dominant mechanism for spatial energy amplification closely resembles that underlying temporal transient growth of perturbation energy in wall-bounded shear flows (including swept Hiemenz flow); a direct quantitative comparison of the maximum energy amplification, however, is difficult.

\section{Summary and conclusions}

This study has demonstrated that optimal spatial disturbances in swept Hiemenz flow take the shape of spanwise counter-rotating vortices. Large amplification of perturbation energy between two spanwise locations is observed due to the interaction of spanwise vortices with the basic spanwise shear $W^{\prime}$ which in turn generates spanwise perturbation velocity $w$ via the lift-up mechanism (Landahl 1980). 

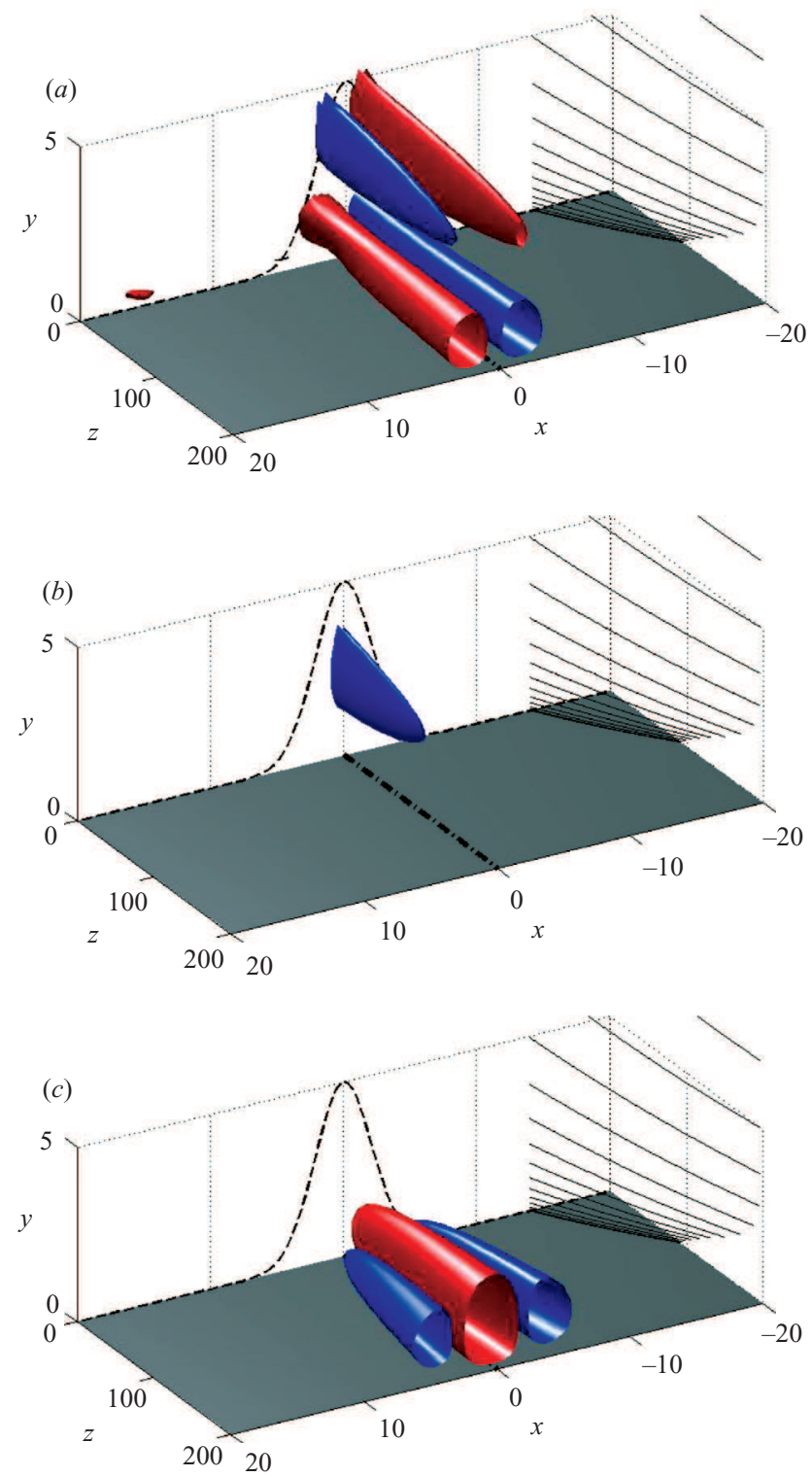

FIGURE 4. Isosurface at $20 \%$ of the maximum chordwise $u$-velocity (a), wall-normal $v$-velocity $(b)$, and spanwise $w$-velocity (c), for an optimal disturbance developing from $z=0$ to $z_{\max }=200$. Blue and red surfaces represent negative and positive values, respectively. The remaining parameters have been set to $R e=550, L=3, \omega=0$. The energy weight function $\lambda_{E}$ is sketched as a dashed line, and streamlines of the basic flow are indicated as lines on the right-hand side of each plot.

In view of the Blasius boundary layer, where an optimal transverse wavenumber $\beta$ for the spatial amplication of energy has been found (Andersson et al. 1999; Luchini 2000; Tumin \& Reshotko 2001), we expect a similar lift-up mechanism associated with the spanwise shear $W^{\prime}$ of the swept Hiemenz flow to exhibit an analogous optimal chordwise scaling. Recall that the necessary chordwise weight function $\lambda_{E}(x)$ acts as a filter which limits the chordwise extent of the optimal perturbation and constrains the 

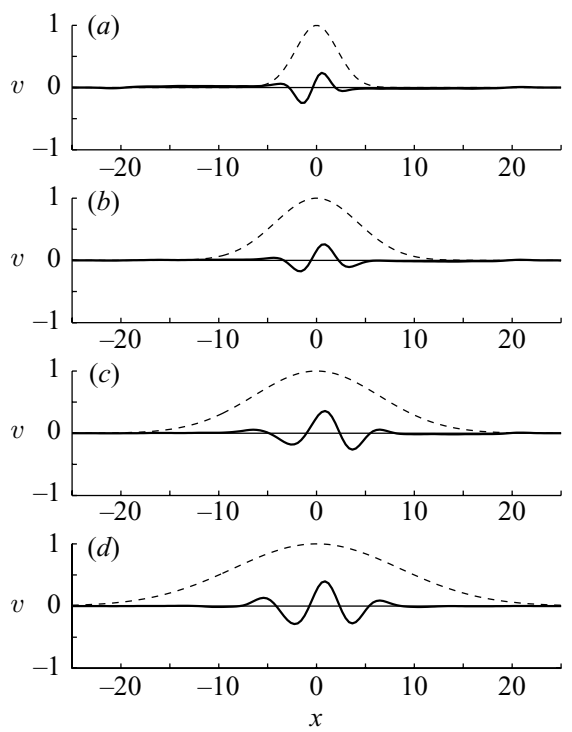

FiguRE 5. Wall-normal $v$-velocity profiles for an optimal perturbation versus chordwise $x$-direction at $z=0$, for chordwise parameter $(a) L=3,(b) 6,(c) 9$ and (d) 12. The flow parameters have been set to $z_{\max }=125, R e=550, \omega=0$. The energy weight function $\lambda_{E}$ is sketched as a thin dashed line.

optimization process. The choice of $L$ also has to reflect the region of validity of the Hiemenz model to accurately resemble the flow field in the vicinity of the stagnation line of a blunt yawed body (Obrist \& Schmid 2003a). As the chordwise parameter $L$ increases, the Gaussian weight function widens and more spanwise vortices are selected as the optimal perturbation (see figure 5).

The basic spanwise velocity component $W$ in swept Hiemenz flow closely resembles the Blasius velocity profile $U_{B L}$ after adjustments have been made regarding the different non-dimensional boundary layer thicknesses, i.e. $\delta=3$ for the swept Hiemenz boundary layer versus $d=5$ for the Blasius profile. Using this adaptation, the optimal streak spacing of $\beta=0.45$ found in Blasius boundary layers can be compared to the chordwise spacing of spanwise Hiemenz streaks. To this end, we extract the chordwise streak spacing $\Lambda$ from our computations at $z=z_{\max }$ and construct an equivalent local chordwise wavenumber $\gamma=(2 \pi / \Lambda) \times(d / \delta)$. As the width $L$ of the Gaussian weight function increases, allowing more initial spanwise vortices to generate spanwise streaks further downstream, the equivalent local chordwise wavenumber $\gamma$ approaches the optimal value of 0.45 found for the Blasius profile.

Taking into account this scaling as well as the optimal perturbation dynamics presented above, we conclude that, despite significant differences in the basic flow profiles, for sweep Reynolds numbers higher than $R e \sim 10^{2}$ the spatial characteristics of swept Hiemenz flow closely resemble the dynamic features found in twodimensional Blasius boundary layers: optimal spatial perturbations consist of steady counter-rotating spanwise vortices which further downstream develop into highly energetic spanwise streaks via the lift-up mechanism, with their chordwise spacing being similar to that found in Blasius boundary layers (once the appropriate scaling adjustments have been made). 
The support of Airbus Industries and Délégation Générale pour l'Armement is gratefully acknowledged.

\section{REFERENCES}

Andersson, P., Berggren, M. \& Henningson, D. S. 1999 Optimal disturbances and bypass transition in boundary layers. Phys. Fluids 11, 134-150.

Bertolotti, F. P., Herbert, T. \& Spalart, P. R. 1992 Linear and nonlinear stability of the Blasius boundary layer. J. Fluid Mech. 242, 441-474.

Chang, C. L. \& Malik, M. R. 1993 Non-parallel stability of compressible boundary layers. AIAA Paper 93-2912.

Cumpsty, N. A. \& Head, M. R. 1969 The calculation of the three-dimensional turbulent boundary layer. Part III: Comparison of attachment-line calculations with experiment. Aero. Q. 20, 99-184.

Gaster, M. 1967 On the flow along swept leading edges. Aero. Q. 18, 165-184.

GöRTLER, H. 1955 Dreidimensionale Instabilität der ebenen Staupunktsströmung gegenüber wirbelartigen Störungen. In 50 Jahre Grenzschichtforschung (ed. H. Görtler \& W. Tollmien). Vieweg, Braunschweig.

Gregory, M. A. 1960 Transition and the spread of turbulence on a $60^{\circ}$ swept-back wing. $J$. $R$. Aero. Soc. 64, 562.

GuÉGAN, A. 2007 Optimal perturbations in swept leading-edge boundary layers. PhD thesis, Ecole Polytechnique, Palaiseau, France.

GuÉGan, A., Huerre, P. \& SCHMID, P. J. 2007 Optimal disturbances in swept Hiemenz flow. J. Fluid Mech. 578, 223-232.

GuÉgan, A., Schmid, P. J. \& Huerre, P. 2006 Optimal energy growth and optimal control in swept Hiemenz flow. J. Fluid Mech. 566, 11-45.

Hall, P., Malik, M. R. \& Poll, D. I. A. 1984 On the stability of an infinite swept attachment line boundary layer. Proc. R. Soc. Lond. A 395, 229-245.

Hämmerlin, G. 1955 Zur Instabilitätstheorie der ebenen Staupunktsströmung. In 50 Jahre Grenzschichtforschung (ed. H. Görtler \& W. Tollmien). Vieweg, Braunschweig.

JosLIN, R. D. 1995 Direct simulation of evolution and control of three-dimensional instabilities in attachment-line boundary layers. J. Fluid Mech. 291, 369-392.

LANDAHL, M. T. 1980 A note on an algebraic instability of inviscid parallel shear flows. $J$. Fluid Mech. 98, 243-251.

Lin, R.-S. \& MaliK, M. R. 1996 On the stability of attachment-line boundary layers. Part 1. The incompressible swept Hiemenz flow. J. Fluid Mech. 311, 239-255.

LUCHINI, P. 2000 Reynolds-number-independent instability of the boundary layer over a flat surface: optimal perturbations. J. Fluid Mech. 404, 289-309.

Obrist, D. \& Schmid, P. J. $2003 a$ On the linear stability of swept attachment-line boundary layer flow. Part 1. Spectrum and asymptotic behaviour. J. Fluid Mech. 493, 1-29.

Obrist, D. \& Schmid, P. J. $2003 b$ On the linear stability of swept attachment-line boundary layer flow. Part 2. Non-modal effects and receptivity. J. Fluid Mech. 493, 31-58.

Pfenninger, W. 1977 Laminar flow control - Laminarization. Special Course on Concepts for Drag Reduction. Tech. Rep. 654. AGARD.

Pfenninger, W. \& BACON, J. W. 1969 Amplified laminar boundary layer oscillations and transition at the front attachment line of a $45 \mathrm{deg}$ swept flat-nosed wing with and without suction. In Viscous drag reduction (ed. C. S. Wells), pp. 85-105. Plenum.

Theofilis, V. 1995 Spatial stability of incompressible attachment-line flow. Theor. Comput. Fluid Dyn. 7, 159-171.

Theofilis, V. 1998 On linear and nonlinear instability of the incompressible swept attachment-line boundary layer. J. Fluid Mech. 355, 193-227.

Tumin, A. \& Reshotкo, E. 2001 Spatial theory of optimal disturbances in boundary layers. Phys. Fluids 13, 2097-2104.

TurkyilmazoG̃Lu, M. \& GaJjar, J. S. B. 1999 On the absolute instability of the attachment-line and swept Hiemenz boundary layers. Theor. Comput. Fluid Dyn. 13, 57-75. 